TECHNICAL NOTE

\author{
M.D. Hope \\ D.D. Purcell \\ T.A. Hope \\ C. von Morze \\ D.B. Vigneron \\ M.T. Alley \\ W.P. Dillon
}

\section{Complete Intracranial Arterial and Venous Blood Flow Evaluation with 4D Flow MR Imaging}

SUMMARY: Time-resolved, 3D velocity-encoded MR imaging (4D Flow) allows for the acquisition of dynamic, multidirectional data on blood flow and has recently been used for the evaluation of intracranial arterial flow. Using a 3T system with optimization of both temporal resolution and $k$-space subsampling with a combination of parallel imaging and cut-corner acquisition, we present the clinical assessment of a patient with an arteriovenous malformation by providing complete intracranial arterial and venous coverage in a reasonable scan time.
$\mathbf{P}$ hase-contrast MR imaging enables the acquisition of multidirectional blood velocity data. With time-resolved, 3D phase-contrast MR imaging (4D Flow), electrocardiographic gating is used to create cine volumetric data. The technique has been most extensively used for the evaluation of patterns of blood flow in the thoracic aorta, including characterization of abnormal flow patterns associated with pathologic disorders such as ascending aortic aneurysm and dissection. ${ }^{1,2}$ Recent studies have explored the use of $4 \mathrm{D}$ Flow imaging for other areas of vascular anatomy and pathology, including intracranial arterial blood flow. ${ }^{3-5}$ We have furthered these efforts toward clinical neurovascular imaging by modifying the $4 \mathrm{D}$ Flow technique with the goal of complete intracranial arterial and venous coverage to provide clinical assessment of a patient with an arteriovenous malformation (AVM).

Detailed evaluation of the arterial inflow and venous drainage of AVMs is important for clinical evaluation and management. ${ }^{6}$ The use of $4 \mathrm{D}$ Flow may aid characterization of the pattern and distribution of feeding arteries by quantification of the relative blood flow through vessels of interest. In a similar fashion, 4D Flow could be useful for the evaluation of venous anatomy, with the additional benefit of identification of venous stenoses as regions of local acceleration of venous blood flow as well as areas at risk for wall shear stress-induced pathologic processes. Stenosis of high-flow draining veins is thought to induce a redistribution of blood flow within an AVM nidus, causing a hemodynamic overload and, consequently, an increased risk for rupture. ${ }^{7}$ In addition, $4 \mathrm{D}$ Flow may be of benefit in conjunction with endovascular procedures or stereotactic radiation to assess changes in relative blood flow entering and exiting an AVM status postintervention. Previous work with animal models has demonstrated that arterial characteristics of venous drainage from AVMs, including pulsatility, high peak velocities, and a larger difference between maximum and minimum velocities, are significantly reduced after successful intervention. ${ }^{8}$

Lengthy scan time has been a limitation of neurovascular

Received January 22, 2008; accepted after revision March 23

From the Department of Radiology (M.D.H., D.D.P., T.A.H., C.v.M., D.B.V., W.P.D.), University of California, San Francisco, Calif, and Department of Radiology (M.T.A.), Stanford University School of Medicine, Stanford, Calif.

Please address correspondence to Michael D. Hope, MD, Department of Radiology, 505 Parnassus Ave, Box 0628, University of California at San Francisco, San Francisco, CA 94143-0628; e-mail: mdhope@stanfordalumni.org

DOI 10.3174/ajnr.A1138
4D Flow. Even with the limited spatial acquisitions described in previous studies (ie, slab thickness less than $5 \mathrm{~cm}$ ), scan times often exceeded 20 minutes. Given our goal of a clinically feasible whole-brain arterial and venous 4D Flow acquisition, the reduction of scan time was of central importance. We have optimized the 3 possibilities available to us to limit scan time: reducing temporal and spatial resolution and subsampling $k$-space.

We present whole-brain arterial and venous neurovascular 4D Flow datasets visualized with streamlines and vector fields in both healthy subjects and a patient with AVM. This study is one of the first to clinically assess significant in vivo neurovascular pathologic disorders and evaluate intracranial venous flow with the 4D Flow technique.

\section{Technique}

Time-resolved, 3D velocity-encoded MR imaging was used to assess intracranial blood flow in 2 healthy subjects and 1 patient with a large left frontoparietal AVM. Our institutional review board approved all studies, and we obtained informed consent from all participants.

The technique has been validated previously. ${ }^{9} \mathrm{D}$ anatomic images were acquired simultaneously with 3 directional velocity fields. Measurements were retrospectively gated to the electrocardiographic cycle. All measurements were performed on a 3T system (Signa CV/i, GE Healthcare, Milwaukee, Wis; Gmax, $40 \mathrm{mT} / \mathrm{m}$; rise time, 268 $\mu \mathrm{sec})$. Scans were performed with an 8-channel head coil with the following imaging parameters: fractional FOV, $180 \times 162 \mathrm{~mm}^{2}$; slab thickness, $128 \mathrm{~mm}$; matrix, $300 \times 162 \times 80$; effective spatial resolution, $0.6 \times 1.0 \times 1.6 \mathrm{~mm}^{3}$; interpolated spatial resolution, $0.35 \times$ $0.35 \times 1.6 \mathrm{~mm}^{3}$. Eight $(\mathrm{ky}, \mathrm{kz})$ phase encode pairs were repeated in each RR interval, resulting in a temporal resolution of 176 to $217 \mathrm{~ms}$. For each subject, studies were performed with 2 velocity encoding values, 1 appropriate for arterial evaluation, the other for venous: 100 and $25 \mathrm{~cm} / \mathrm{s}$, respectively, for the healthy subjects, 200 and $50 \mathrm{~cm} / \mathrm{s}$, respectively, for the patient. These values were selected on the basis of previously reported data on intracranial blood-flow velocity and the results of our own preliminary scans. ${ }^{3-5}$ Given our goal of characterizing the arterial inflow and venous drainage of AVMs, with evaluation of vessels as small as 2 to $3 \mathrm{~mm}$ in diameter, we were limited in our ability to reduce spatial resolution. Generalized autocalibrating partially parallel acquisition (GRAPPA) with an acceleration factor of 2 and a cut-corner acquisition of $k$-space was used. The area of the remaining $k$-space data in this "cut-corner" acquisition is defined by the ellipse $\Pi \mathrm{K}_{\mathrm{zmax}} \mathrm{K}_{\mathrm{ymax}}{ }^{10}{ }^{10} \mathrm{~A}$ total of 677 heartbeats were required for data acquisition, resulting in scan times of 7 to 12 minutes. 

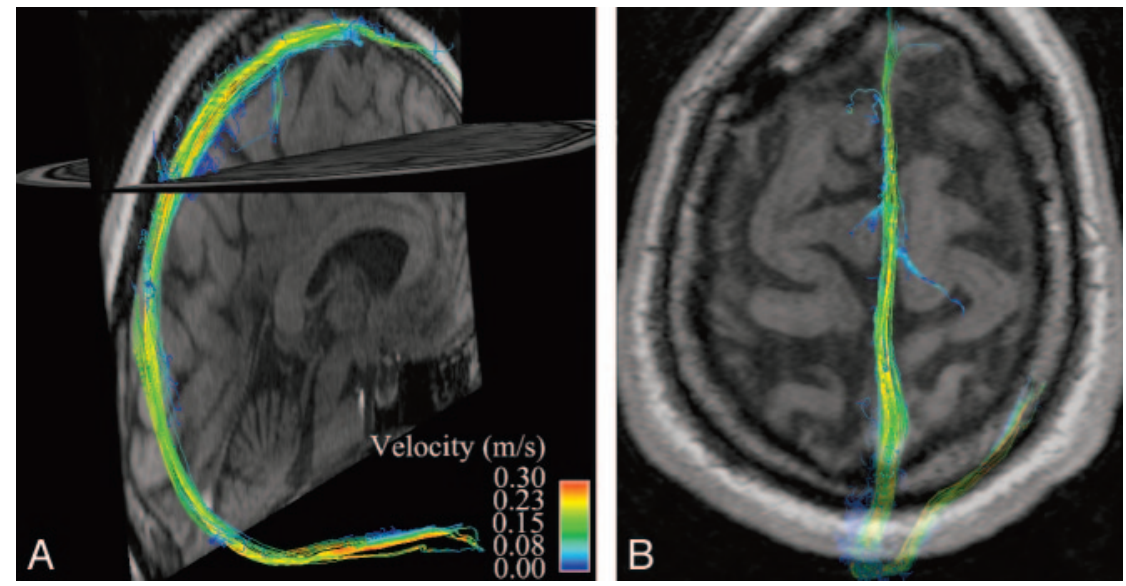

Fig 1. Whole-brain venous imaging in a healthy volunteer. A maps streamlines of venous blood flow in the superior sagittal and right transverse sinuses onto a midline sagittal magnitude image. Streamlines are imaginary lines aligned with local vector fields and represent the flow field at a given moment in the cardiac cycle. They are color coded for velocity. $B$ demonstrates flow in the superior sagittal sinus at the vertex with a semitransparent axial magnitude image provided for orientation.
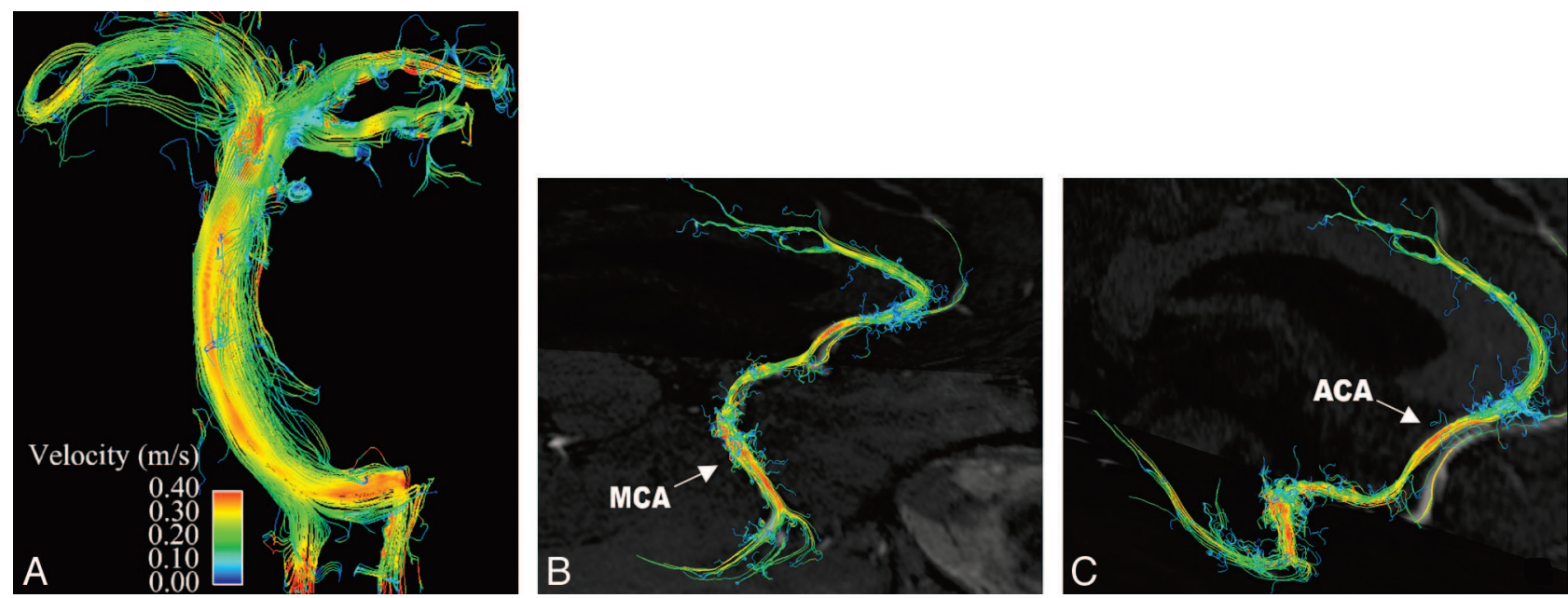

Fig 2. Whole-brain arterial imaging in a healthy volunteer. $A$ depicts midsystolic blood flow with streamlines in the basilar artery, with flow in the bilateral vertebral arteries seen inferiorly and flow in the posterior cerebral artery seen superiorly. In addition, flow within the bilateral superior cerebellar arteries can be appreciated. $B$ and $C$ demonstrate flow in the right anterior and middle cerebral arteries. The pericallosal branch of the anterior cerebral artery is well visualized, as is the middle cerebral artery trifurcation. Magnitude images are provided in midline sagittal and axial planes for orientation.

Data were corrected for Maxwell phase effects, encoding errors as a result of the gradient field distortions and effects from eddy currents. ${ }^{1-13}$ Corrected velocity data were imported into 3D visualization software (EnSight; CEI, Apex, NC). This software program enables the visualization of complex $3 \mathrm{D}$ and $4 \mathrm{D}$ datasets by providing a variety of data manipulation tools including $2 \mathrm{D}$ velocity vector fields mapped onto planes of interest, 3D streamlines, and particle traces. Planes of data perpendicular to vessels of interest in both healthy subjects and the patient were then extracted for quantification of relative blood flow with a proprietary software program (Aspire2; Stanford University, Stanford, Calif).

\section{Results}

We have demonstrated normal venous and arterial blood flow in healthy subjects, with clear visualization of flow in the dural venous sinuses (Fig 1) and the anterior and posterior intracranial arterial circulation (Fig 2). In a patient with a large left frontoparietal AVM, we have visualized and quantified a marked increase in venous flow through the superior sagittal sinus (5.1 times greater) and superficial veins compared with healthy subjects. Time-resolved analysis of venous drainage from the AVM revealed arterial pulsatility to the venous flow (Fig 3).

We have quantified relative blood flow in the 3 largest arteries supplying the AVM and demonstrated a marked increase in flow (3.3 times greater) through the ipsilateral internal carotid artery compared with healthy subjects (Fig 4). In addition, we have demonstrated somewhat disorganized, circulating flow within the AVM nidus (Fig 5). Streamline evaluation of arterial inflow to the AVM was limited by vessel tortuosity and resulting disorganized flow but did reveal many regions of locally increased velocity within the branches of the anterior and middle cerebral arteries supplying the AVM (Fig $6)$.

\section{Discussion}

The use of 4D Flow MR imaging is an effective means to evaluate multidirectional blood flow of intracranial vessels. We have successfully used a modification of the technique for whole-brain arterial and venous imaging in healthy subjects 

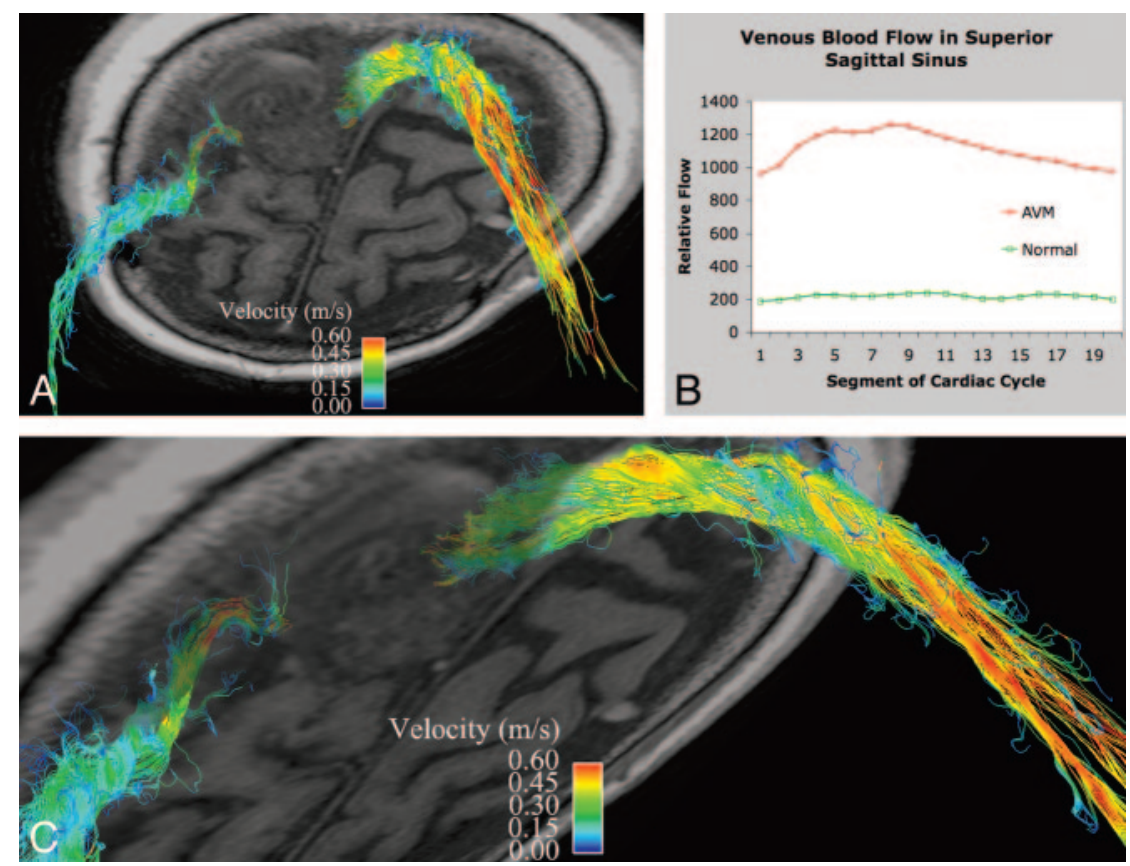

Fig 3. Venous drainage in a patient with an AVM. $A$ is a slightly oblique view of an axial section through the left frontoparietal AVM with streamlines overlaid to depict venous flow in the superior sagittal sinus and a right superficial vein. $B$ shows the marked increase in blood flow in the superior sagittal sinus in the patient compared with a healthy subject (5.1 times greater). Note the arterial pulsatility of the venous drainage. $C$ is a magnified oblique view of the high-velocity venous drainage from the AVM.
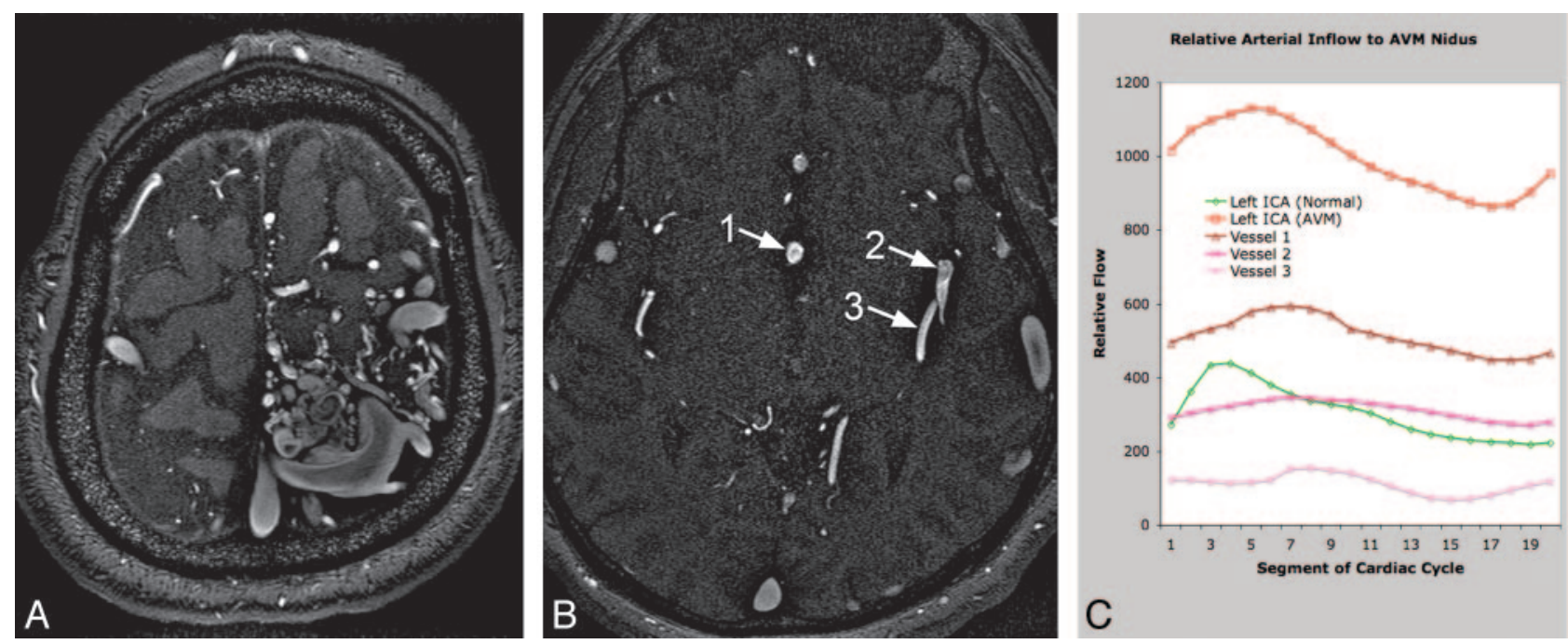

Fig 4. Quantification of arterial inflow to AVM nidus. $A$ is an axial section from a time-of-flight MRA demonstrating the left frontoparietal nidus. $B$ has singled out the 3 largest arteries supplying the nidus: vessel 1 is an anomalous branch of the anterior cerebral artery running along the septum pellucidum, and vessels 2 and 3 are posterior left middle cerebral artery branches. $C$ shows the marked increase in blood flow through the left internal carotid artery in the patient compared with a healthy subject (3.3 times greater), as well as the relative arterial contribution to the AVM nidus of the blood vessels labeled on the MRA.

and in a patient with an AVM. By using a lower temporal resolution than previous neurovascular 4D Flow studies and a combination of parallel imaging and a cut-corner acquisition of $k$-space, we have achieved a clinically reasonable scan time of 677 heartbeats ( or less than 10 minutes at heart rates greater than 68 beats per minute).

Bammer et $\mathrm{al}^{4}$ have described the effects of lower temporal resolution on $4 \mathrm{D}$ Flow velocity data at $3 \mathrm{~T}$. At the lower temporal resolution that we have used (approximately $200 \mathrm{~ms}$ ), there is an underestimation of peak velocities and a broadening of the systolic phase, which may be of significance if calculation of resistive and pulsatility indexes, precise calculation of blood flow, or estimation of secondary blood-flow parameters such as shear stress were of clinical importance. They have also assessed the effects of parallel imaging with different GRAPPA acceleration factors and demonstrated relative preservation of mean velocity and flow data with an acceleration factor of $3 .^{4}$

In addition, we used another approach to subsampling $k$ space: the exclusion of the corners of $k$-space, which can reduce scan time by up to $25 \%$ and can be used in combination with parallel imaging. The rationale for this approach stems from work by Markl and Hennig, ${ }^{14}$ which demonstrated that velocity-induced phase shifts are encoded mainly in central $k$-space. Evaluation of a limited number of $4 \mathrm{D}$ Flow datasets 

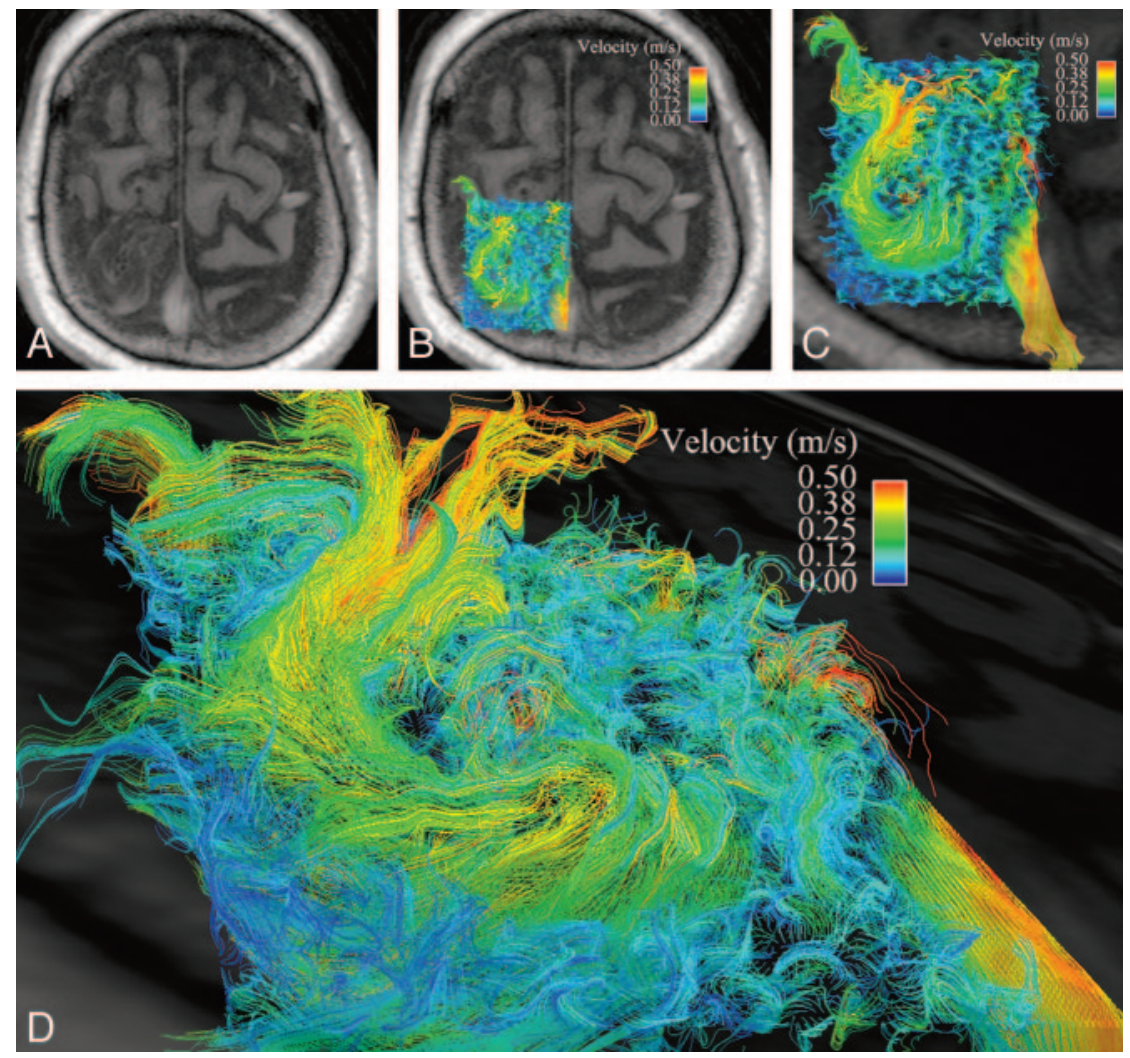

Fig 5. Disorganized, circulating blood flow within the AVM nidus. $A$ is an axial magnitude section through the large left frontoparietal AVM. $B$ has velocity data overlaid on this axial section $C$ is a magnified view of the AVM nidus with $3 D$ velocity data from midsystole, as represented as a curved vector field, demonstrating somewhat disorganized, circulating flow. Note the high-velocity venous drainage in the superior sagittal sinus, which can be visualized through the semitransparent axial section. $D$ is an oblique view at higher magnification.
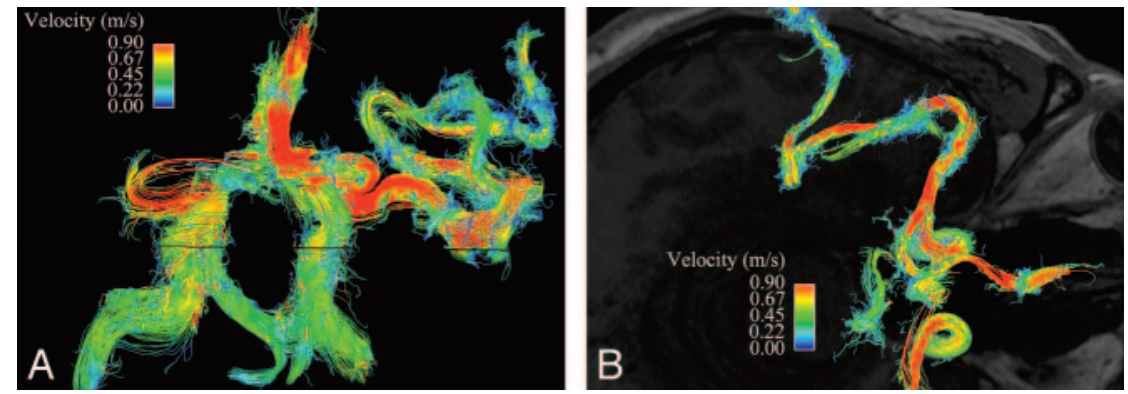

Fig 6. Arterial inflow to the AVM. $A$ is a streamline representation of the anterior and posterior intracranial circulation in an anteroposterior projection, with interrogation of the left middle cerebral artery branches leading to the AVM nidus. B overlays the same velocity data on an axial magnitude section at the level of the internal carotid arteries. Despite the marked tortuosity of these vessels and resulting disorganized flow, which limits streamline evaluation, regions of locally increased velocity within arterial feeders are easily identified and may help to predict the development of flow-related extranidal arterial aneurysms.

obtained without the corners of $k$-space demonstrates good preservation of velocity data by qualitative and quantitative assessment. Although more extensive analysis needs to be done for validation, the qualitative assessment of arterial and venous blood flow and quantification of relative flow in vessels of interest that we present may be of clinical use for neurovascular evaluation.

By providing relative flow quantification in feeding arteries seen by MR angiography, 4D Flow can augment the standard evaluation of arterial inflow for AVMs. This method could be of clinical use for preprocedural planning and assessment status postintervention. Evaluation of venous drainage may also be important in the evaluation of posttreatment status because decreased flow in draining veins and absence of arterial pulsatility would be consistent with successful intervention. Fur- thermore, characterization of complex flow features within an AVM nidus may prove useful for estimation of the relative risk for rupture, and identification of regions of locally increased velocity within arterial feeders may help to predict the development of flow-related extranidal arterial aneurysms, which have been reported in $7 \%$ to $20 \%$ of cases. ${ }^{15}$

\section{Conclusions}

The use of 4D Flow has the potential to develop into a clinically useful tool for the evaluation of intracranial vasculature. With its detailed characterization of complex, dynamic blood-flow patterns and its ability to quantify flow, the technique could supplement both current noninvasive and invasive imaging of intracranial vascular pathologic disorders, including AVM, as we have investigated. Analysis of intracranial venous flow has 
not been reported previously and could become an important clinical indication for 4D Flow. In AVMs, identification of venous stenoses and confirmation of decreased arterial characteristics of venous drainage status postintervention may better inform clinical management. In a more broad sense, $4 \mathrm{D}$ Flow could be used as a noncontrast means of evaluation of dural venous sinus pathologic processes, including dural sinus thrombosis.

\section{References}

1. Markl M, Draney MT, Hope MD, et al. Time-resolved 3-dimensional velocity mapping in the thoracic aorta: visualization of 3-directional blood flow patterns in healthy volunteers and patients. J Comput Assist Tomogr 2004;28:459-68

2. Hope TA, Markl M, Wigström L, et al. A comparison of flow patterns in patients with ascending aortic aneurysms and volunteers using four-dimensional magnetic resonance velocity imaging. J Magn Reson Imaging 2007;26:1471-79

3. Yamashita S, Isoda $H$, Hirano $M$, et al. Visualization of hemodynamics in intracranial arteries using time-resolved three-dimensional phase-contrast MRI. J Magn Reson Imaging 2007;25:473-78

4. Bammer R, Hope TA, Aksoy M, et al. Time-resolved 3D quantitative flow MRI of the major intracranial vessels: initial experience and comparative evaluation at $1.5 \mathrm{~T}$ and $3.0 \mathrm{~T}$ in combination with parallel imaging. Magn Reson Med 2007;57:127-40

5. Wetzel S, Meckel S, Frydrychowicz A, et al. In vivo assessment and visualiza- tion of intracranial arterial hemodynamics with flow-sensitized 4D MR imaging at 3T. AJNR Am J Neuroradiol 2007;28:433-38

6. Ledezma CJ, Hoh BL, Carter BS, et al. Complications of cerebral arteriovenous malformation embolization: multivariate analysis of predictive factors. $\mathrm{Neu}$ rosurgery 2006;58:602-11

7. Hademenos GJ, Massoud TF. Risk of intracranial arteriovenous malformation rupture due to venous drainage impairment. A theoretical analysis. Stroke 1996;27:1072-83

8. Murayama Y, Massoud TF, Viñuela F. Transvenous hemodynamic assessment of experimental arteriovenous malformations. Doppler guidewire monitoring of embolotherapy in a swine model. Stroke 1996;27:1365-72

9. Markl M, Chan FP, Alley MT, et al. Time-resolved three-dimensional phasecontrast MRI. J Magn Reson Imaging 2003;17:499-506

10. Bernstein MA, Fain SB, Riederer SJ. Effect of windowing and zero-filled reconstruction of MRI data on spatial resolution and acquisition strategy. J Magn Reson Imaging 2001;14:270-80

11. Bernstein MA, Zhou XJ, Polzin JA, et al. Concomitant gradient terms in phase contrast MR: analysis and correction. Magn Reson Med 1998;39:300-08

12. Markl M, Bammer R, Alley MT, et al. Generalized reconstruction of phase contrast MRI: analysis and correction of the effect of gradient field distortions. Magn Reson Med 2003;50:791-801

13. Walker PG, Cranney GB, Scheidegger MB, et al. Semiautomated method for noise reduction and background phase error correction in MR phase velocity data. J Magn Reson Imaging 1993;3:521-30

14. Markl M, Hennig J. Phase contrast MRI with improved temporal resolution by view sharing: $\mathrm{k}$-space related velocity mapping properties. Magn Reson Imaging 2001;19:669-76

15. Kim EJ, Halim AX, Dowd CF, et al. The relationship of coexisting extranidal aneurysms to intracranial hemorrhage in patients harboring brain arteriovenous malformations. Neurosurgery 2004;54:1349-57 